\title{
Microfiber-knot-resonator-induced energy transferring from vector noise-like pulse to scalar soliton rains in an erbium-doped fiber laser
}

\author{
Jian Zhou, Shuchang Gu, Zikai Chen, Bowen Lou, Yuyuan Jiang, Junqing Zhao, Lei Li, Dingyuan \\ Tang, Deyuan Shen, Lei Su, and Luming Zhao, Senior Member, IEEE
}

\begin{abstract}
We report on scalar soliton rains (SRs) shedding from vector square-wave noise-like pulse (NLP) by introducing a microfiber-knot-resonator (MKR) into an erbium-doped fiber (EDF) laser. The EDF laser has an ultralong cavity of $\sim 3.07 \mathrm{~km}$, resulting in a large net anomalous-dispersion, which facilitates the formation of NLP. Once an MKR was further incorporated, comblike filtering effect could be clearly observed on the emission spectrum. Apart from that, SRs shedding from the NLP were observed, which resulted from the MKR-induced energy transferring effect from the NLP to SRs. It was further found that the SRs existed only along a particular polarization direction, whereas the NLPs could be found in any pair of orthogonal polarization directions, despite that the peak power might be different depending on polarization. These suggest that the SRs possess scalar feature, whereas the co-circulating NLP is a package of vector pulses. Our results represent the first observation of MKR-induced pulse energy transferring effect and also the first observation of the co-existence of scalar and vector pulses in a single optical system. The related dynamics and mechanisms are analyzed in detail. Besides enriching the dynamics and interactions of optical pulses in fiber lasers, our results extend the applications of MKR to the area of pulse manipulations through spectral tailoring and local nonlinearity engineering.
\end{abstract}

Index Terms-Optical pulses, Optical resonators, Optical fiber devices, Optical fiber lasers, Optical signal processing.

\section{INTRODUCTION}

$\mathrm{R}$ ELYING on rich physical mechanisms and complex dynamics in passively mode-locked fiber lasers, various optical pulses, as well as the related interactions and dynamics, have been explored extensively in past decades. These are still among the hot topics to date, due to the further in-depth studies and the applications of some newly-emerging

\section{Manuscript received.}

This work was supported in part by the National Natural Science Foundation of China (NSFC) under Grants 61705094, 11674133 and 61575089, in part by the Natural Science Foundation of Jiangsu Province under Grant BK20170243, in part by the Royal Society under Grant IE161214, and in part by the European Union's Horizon 2020 research and innovation programme under the Marie Skłodowska-Curie Grant 790666. (Corresponding author: Junqing Zhao and Luming Zhao)

J. Zhou, S. Gu, Z. Chen, B. Lou, Y. Jiang, J. Zhao, L. Li, and D. Shen are with the Jiangsu Key Laboratory of Advanced Laser Materials and Devices, Jiangsu Collaborative Innovation Center of Advanced Laser Technology and Emerging Industry, School of Physics and Electronic Engineering, Jiangsu Normal University, Xuzhou 221116, Jiangsu, China (e-mail: 835139040@qq.com; 1272640745@qq.com;1002886839@qq.com; materials as saturable absorbers (SAs), especially the intensively investigated two-dimensional (2D) materials $[1,2]$. Besides several early discovered pulse types, such as conventional solitons, dispersion-managed solitons, dissipative solitons, etc., in recent years, particular attentions have been paid to some long-duration, wave-breaking-free pulses that are formed typically in long-cavity fiber lasers, where considerable accumulatively nonlinear effects can typically be developed due to strong field-confinement of single mode fiber (SMF) and the considerable interaction length. With such a cavity, multiple solitons would be generated due to the well-known areatheorem if the net cavity dispersion falls in the anomalous dispersion regime [3]. Even for the dispersion-managed solitons or dissipative solitons, there are still some upper limits on the single pulse energies and energy quantization still exists [4]-[6].

However, in some cases where a type of peak power clamping (PPC) effect dominates, i.e., the pulse amplitude can be fixed at a roughly constant value. As a remarkable consequence, both the pulse duration and energy can scale with the incident pump power to some unprecedented levels before wave-breaking occurring, if the operation is properly manipulated. So-called dissipative soliton resonance (DSR) is just the most representative regime [7]-[9]. However, not all the square wave or rectangular-shaped pulses can be attributed to such a regime. Detailed studies found that some of them were actually a type of noise-like pulses (NLPs). Although an NLP always shows a rectangular envelop on an oscilloscope, autocorrelation (AC) measurement suggest that it is in fact a tight bunch of many ultrashort pulses [10]-[15].

Despite the lengthy-fiber-induced strong global intra-cavity nonlinearity, some highly nonlinear fiber devices can induce abruptly considerable change on the local nonlinearity. Among them, one interesting device is so-called microfiber knot

1962264982@qq.com; 1461673357@qq.com; junqingzhao@outlook.com; Leelei@jsnu.edu.cn; shendy@fudan.edu.cn).

D. Tang is with the School of Electrical and Electronic Engineering, Nanyang Technological University, Singapore (e-mail: edytang@ntu.edu.sg).

L. Su is with the School of Engineering and Materials Science, Queen Mary University of London, London, UK (email: 1.su@qmul.ac.uk).

L. Zhao is with the Jiangsu Key Laboratory of Advanced Laser Materials and Devices, Jiangsu Collaborative Innovation Center of Advanced Laser Technology and Emerging Industry, School of Physics and Electronic Engineering, Jiangsu Normal University, Xuzhou 221116, China, and also with the School of Engineering and Materials Science, Queen Mary University of London, London, UK (email: 1mzhao@ieee.org).

Copyright (c) 2020 IEEE. 
resonator (MKR) [16]-[19]. Its high nonlinearity results from two aspects. One is that it is an extremely-tapered device, which leads to hundreds of times shrinking in cross section. The second is that it is a resonant device, and a certain portion of the optical field within it can circulate for endless times prior to exiting. One interesting issue is how such a device can modify the pulse dynamics in fiber lasers. Although few earlier reports have found some interesting effects due to its incorporation fiber lasers, those were all realized in short cavities with only weak or even negligible nonlinear effects [20]-[22]. Thus, it is still unknown that how such a device can modify the pulse dynamics in circumstances with strong accumulative nonlinearity. From a more general point, it is to say that how the local nonlinearity exerts influences on the global nonlinearity. For that, in this paper, we investigate the MKRinduced effects in an ultralong erbium-doped fiber (EDF) laser. Without MKR, it is found that the EDF laser can deliver typical NLP, whose duration scales continuously with the incident pump power. Once a house-made MKR is incorporated, initially the NLP still follows similar evolution with the incident pump power. However, it is observed that, some soliton rains (SRs) can shed continuously from the NLP, and meanwhile the NLP maintain a constant duration, when the pump power rises above a threshold-like value. Even more interestingly, the SRs are found to be scalar fields while the NLP is a package of vector fields. That is to say that both scalar and vector fields can co-exist and circulate in a single optical system.

\section{MKR FABRICATION AND THE EDF LASER CONFIGURATION}

The used MKR is shown in Fig. 1(a), where some red light was launched for visibly showing the transmission characteristics of the MKR. The MKR was built by knotting a house-pulled fiber taper fabricated by using a fiber tapering workstation (IPCS-5000, Idealphotonics Inc.). Figure 1(b) shows the tapering waist of the MKR viewed by using a scanning electron microscope (SEM, X-act, Oxford Instruments, Inc.), which gives a measured diameter of $\sim 1.57$ $\mu \mathrm{m}$. The ultra-thin-tapering MKR enables a strong confinement of optical field and thus high local nonlinearity. In our use, the MKR was suspended in air, which can prevent evanescent wave coupling and thus minimize the transmission loss. As measurement, the transmission loss in air is $\sim 2.43 \mathrm{~dB}$. It was further noted that the transmission of the MKR exhibits polarization-dependent characteristics. This results in a power extinction ratio of $\sim 11.56: 1$, i.e., $\sim 10.63 \mathrm{~dB}$.

Transmission spectrum as shown in Fig. 1(c) was measured by using an ASE source (ASE-FL7004, 1530-1610 nm, FiberLabs Inc.) and an optical spectrum analyzer (OSA, AQ6370C, Yokogawa Test \& Measurement Co., Japan) by setting the resolution at $0.02 \mathrm{~nm}$. Figure 1(c) gives a resonance width of $\sim 0.12 \mathrm{~nm}$ (estimated at the full width at half maxima, FWHM) and a free spectrum range (FSR) of $\sim 0.61 \mathrm{~nm}$. The further calculated Q factor, finesse, and loop circumference of the MKR are $\sim 13000, \sim 5.08$, and $\sim 216.23 \mu \mathrm{m}$, respectively.

As shown in Fig. 1(d), the constructed EDF laser exhibits a typical figure-eight (f-8) structure, which comprises a nonlinear amplifying-loop mirror (NALM), acting as an artificial SA, and a unidirectional ring (UR). In the NALM, a $976 \mathrm{~nm}$ laser diode (LD) is used as the pump source. The pump light is coupled into $\sim 80 \mathrm{~cm}$ EDF through a fused wavelength division multiplexer (WDM). The EDF (EDF80, OFS) has a dispersion of $\sim-48$ $\mathrm{ps} /(\mathrm{nm} \cdot \mathrm{km})$ and peak absorption at $1530 \mathrm{~nm}$ of $\sim 80 \mathrm{~dB} / \mathrm{m}$. A three-paddle fiber polarization controller (FPC), noted as FPC1 , is incorporated to adjust the local polarization state (PS). We further use $\sim 35 \mathrm{~m}$ highly nonlinear fiber (HNLF) and $\sim 3021 \mathrm{~m}$ SMF-28e in the NALM to enhance the accumulative cavity nonlinearity. The HNLF has a core diameter of $\sim 3.55 \mu \mathrm{m}$ and dispersion of $\sim 1.31 \mathrm{ps} /(\mathrm{nm} \cdot \mathrm{km})$ at $1550 \mathrm{~nm}$.

The NALM is connected to the UR through a 3-dB fiber optical coupler (FOC). Unidirectional propagation is enabled by using a polarization-independent isolator (PI-ISO). A 20/80 FOC provides the laser output. The second FPC, i.e., FPC-2, is used to further adjust the local PS in the UR. Except the EDF and HNLF, all other fiber pieces in the EDF laser are standard SMF-28e. The total cavity length is $\sim 3072.8 \mathrm{~m}$, resulting in a net anomalous dispersion of $\sim-61.9 \mathrm{ps}^{2}$ around $1550 \mathrm{~nm}$.
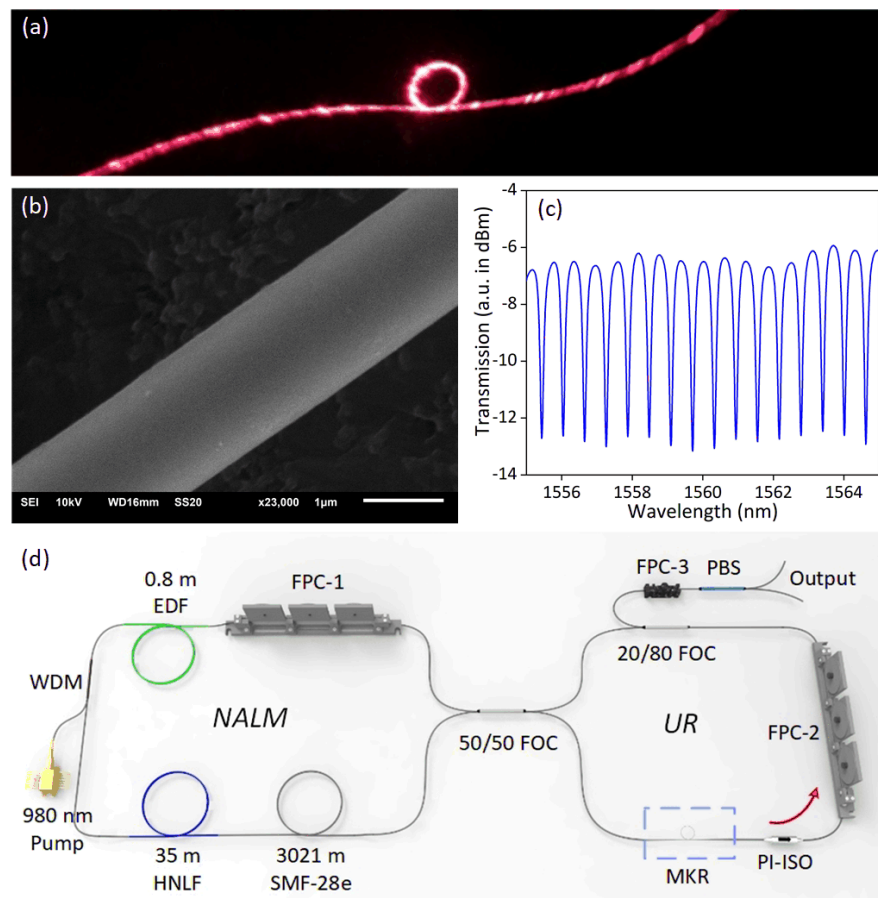

Fig. 1. (a) Photograph of the MKR with propagating red light; (b) SEM around tapering waist of the MKR. (c) transmission spectrum of the MKR. (d) MKRincorporated f-8 EDF fiber laser.

\section{NLP EMISSION CHARACTERISTICS WITHOUT MKR}

At first, we carried out an experiment without incorporation of the MKR. A $63 \mathrm{GHz}$ high-speed oscilloscope (DSO-X 96204Q, Keysight Technologies, Inc.) along with a $45-\mathrm{GHz}$ ultrafast-response photodetector (1014, New Focus, Inc.) were employed to real-time monitor the output pulse as fast as possible. Square-wave-shaped pulse could be achieved by adjusting the two FPCs when the pump power increased to $\sim 30.2 \mathrm{~mW}$. With a fixed PS, as the pump power increased from $\sim 74.8$ to $\sim 292.9 \mathrm{~mW}$, the pulse duration continuously broadened from $\sim 59.9$ to $\sim 246.8$ ns, as shown in Fig. 2(a).

Figure 2(b) shows a typical optical spectrum with pump power of $\sim 255.1 \mathrm{~mW}$. The emitted peak wavelength was $\sim 1560.36 \mathrm{~nm}$ with a $3-\mathrm{dB}$ bandwidth of $\sim 4.88 \mathrm{~nm}$. Figure 2(c) 
plots the corresponding detailed radio frequency (RF) spectrum around the fundamental pulse repetition rate, which peaks at $\sim 66.38 \mathrm{kHz}$ with signal-to-noise ratio (SNR) of $\sim 47.3 \mathrm{~dB}$. The $\mathrm{RF}$ characteristics through this paper were all measured by using an RF spectrum analyzer (N9320B, Agilent Technologies, Inc) in combination with a photodetector (InGaAs Biased Detector, DET01CFC/M, THORLABS Inc.). The inset of Fig. 2(c) shows an RF trace with $10 \mathrm{MHz}$ span. The RF train and RF spectrum around the pulse repetition rate were recorded with 100 and $10 \mathrm{~Hz}$ resolution bandwidth (RBW), respectively.

Considering that the top part of each rectangular-shaped pulse embedded many dense and random spikes, we suspected that the obtained pulse might be a type of NLP. AC measurements were then taken to check that by using an autocorrelator (FR-103HS, Femtochrome Research, Inc.) assisted with a digital oscilloscope (DSO-X3034A, Agilent Technologies, Inc.). The measured AC trace was plotted in Fig. 2 (d), when the pump power was $\sim 255.1 \mathrm{~mW}$. As shown, there existed a short pulse with sub-picosecond FWHM that stood on a much longer pedestal, which was a signature of NLP [10][14]. This confirmed that our EDF laser was indeed operating in the NLP regime. In general, it is better to use a long fiber cavity for the generation of NLP. Our long fiber cavity can facilitate the accumulation of nonlinearity, which is required by the generation of NLP.
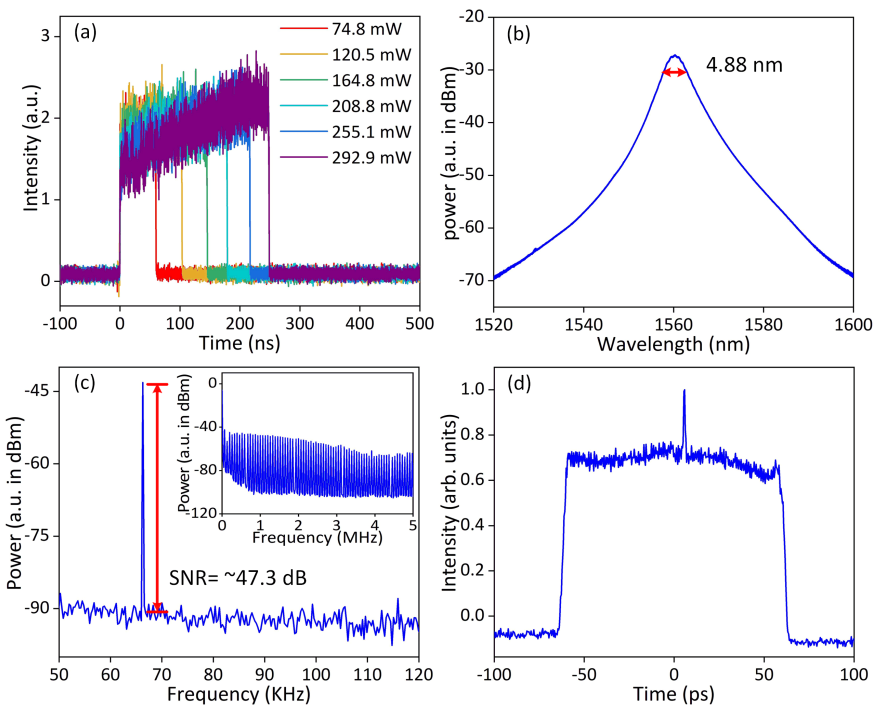

Fig. 2. Square-wave NLP output characteristics without incorporation of the MKR. (a) pulse evolution against pump power; (b) optical spectrum with pump power of $\sim 255.1 \mathrm{~mW}$; the corresponding (c) RF spectrum around the pulse repetition rate; inset: a typical RF train; (d) AC trace.

\section{MKR-INDUCED SOLITON RAIN SHEDDING EFFECTS}

The as-prepared MKR was then incorporated in the UR of the EDF laser, as seen in Fig. 1(d). The cavity was thus elongated by $\sim 0.7 \mathrm{~m}$. Similar to the fiber laser without MKR, the NLP broadened almost linearly with the pump power increasing from $\sim 30.2 \mathrm{~mW}$ to $\sim 164.8 \mathrm{~mW}$. However, an interesting phenomenon occurred when the pump power increased above $\sim 164.8 \mathrm{~mW}$. As seen in Fig. 3(a), the duration of the rectangular NLP was maintained at $\sim 190$ ns when the pump power increased above $\sim 164.8 \mathrm{~mW}$. Instead of further pulse broadening, there were some dense, burst-like pulses continuously shedding from the NLP. The shedding pulses all moved towards one direction i.e. the left on the oscilloscope. Then they vanished. The higher the pump power, the more pulses shed out and the longer the overall span of these pulses from birth to death, i.e., the longer their life time. It could span to $\sim 9.8 \mu \mathrm{s}$ when the pump power increased to the maximum limit of the pump source of $\sim 433.1 \mathrm{~mW}$, which took up a considerable portion of the whole repetition period of $\sim 15.4 \mu \mathrm{s}$, resulting in to a pulse duty circle of $\sim 63.6 \%$. These shedding pulses can be identified as SRs, considering that there are some similar behaviors with earlier reports on SRs [23]-[25]. But some differences still existed since here they were shed and, in fact, converted from a part of the NLP.
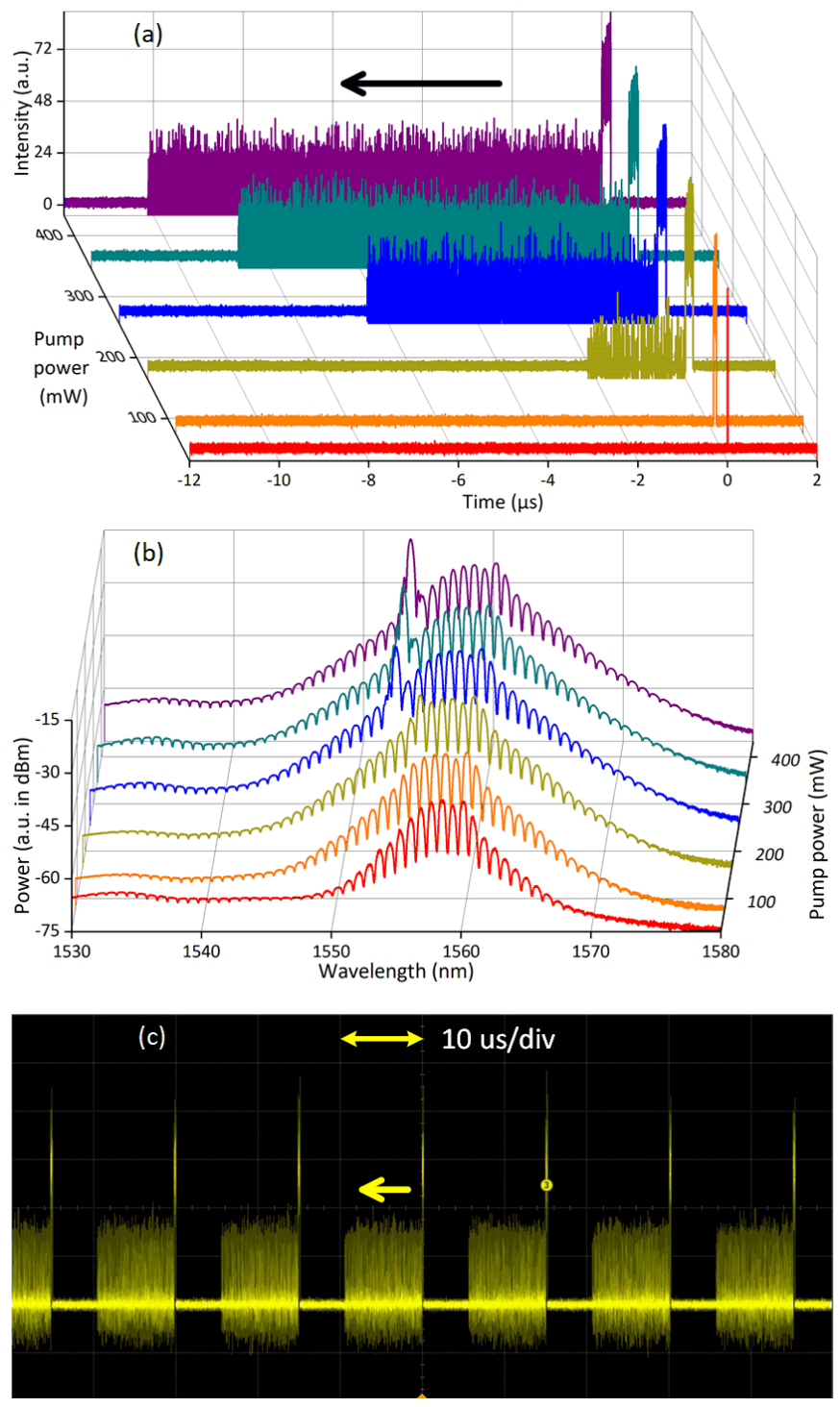

Fig. 3. Pump-power-induced (a) temporal and (b) spectral evolutions; (c) a train of captured NLPs along with shedding SRs when the pump power was $\sim 388.4$ $\mathrm{mW}$.

Figure 3(b) plots the corresponding spectral evolution against pump power. The MKR enabled strong modulations on the spectrum. A strikingly rising spectral peak started to stand out from the main spectral profile when the pump power increased above $\sim 164.8 \mathrm{~mW}$ when the SRs started to shed from the NLP. It was also noted that the spectral peak shifted from $\sim 1556.1$ to $1553.6 \mathrm{~nm}$ as the pump power increased from $\sim 164.8$ to $\sim 433.1$ 
$\mathrm{mW}$. At the maximum pump power, the 3-dB bandwidth of the spectral peak was $\sim 0.37 \mathrm{~nm}$, which is narrower than the FSR of the MKR $(\sim 0.61 \mathrm{~nm})$, probably attributed to the strong rollofffiltering effect of the MKR as measured in Fig. 1(c). Figure 3(c) shows a captured temporal trace with a span of $100 \mu$ s when the pump power was $\sim 388.4 \mathrm{~mW}$.

We also further checked vector characteristics when there were SRs shedding from the NLP. We connected an in-line FPC and a polarization beam splitter (PBS) to the fiber port of the laser output. When the external fiber pigtail's linear birefringence is compensated by the FPC-3, the polarizationresolved characteristics were plotted in Fig. 4. Figure 4(a) show the polarization-resolved temporal characteristics. The rectangular NLP existed in both polarization directions of the PBS, sharing the same pulse duration of $\sim 190 \mathrm{~ns}$, whereas the SRs existed only along the vertical polarization direction. This indicates that the SRs are tightly packaged moving scalar pulses.

Figures 4(b) and 4(c) show the corresponding spectral components along the two orthogonal directions. Both spectral profiles exhibited significant modulations resulted from the comb-like transmission of the MKR. As also noted, the standing-out spectral peak appeared only in one polarizing direction that the SRs just existed.
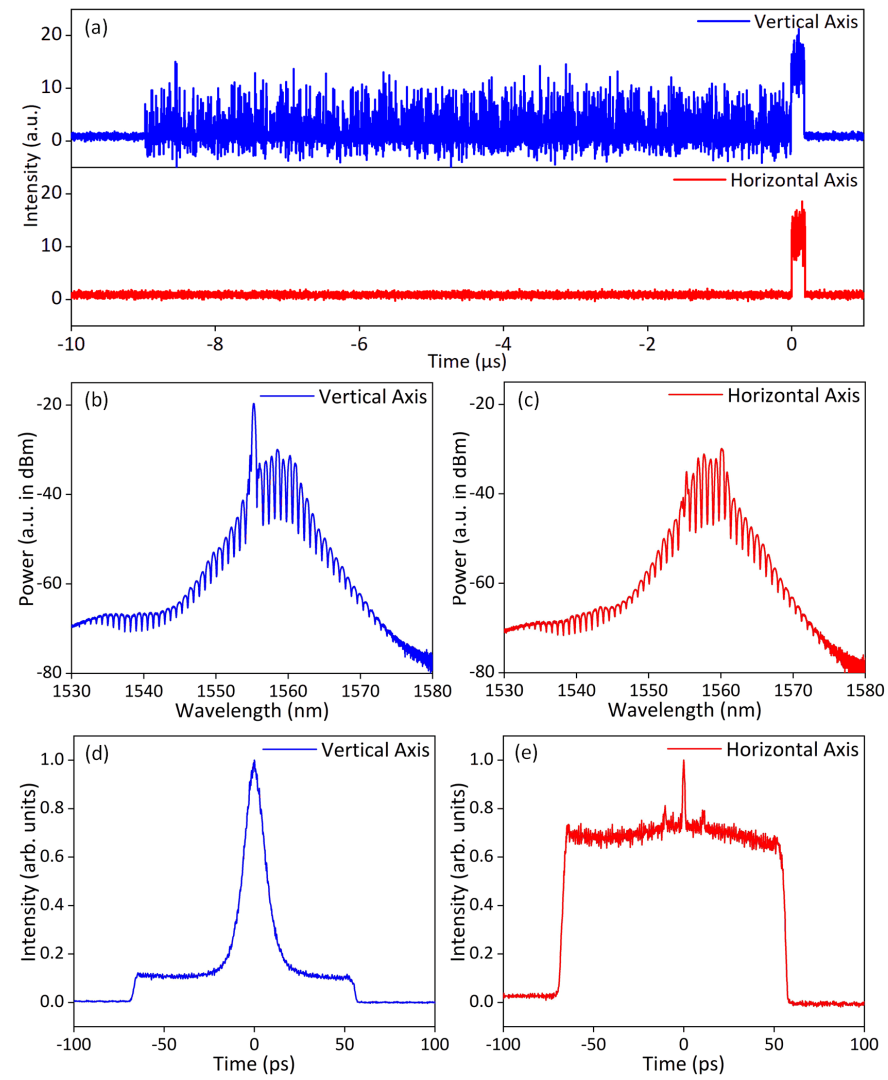

Fig. 4. Polarization-resolved characteristics when there were SRs shedding from the NLP. (a) NLP components in two orthogonal polarizing directions; the corresponding optical spectra [(b) and (c)] and AC traces [(d) and (e)].

The AC trace in the vertical polarization was plotted in Fig. 4(d). A significantly high-rising pulse with an FWHM duration of $\sim 13.6$ ps stood out from a wide and low-intensity pedestal. This indicated that the AC trace should be a measurement result of multiple solitons with similar parameters, or a measurement result of SRs. The horizontally polarized AC trace, as plotted in Fig. 4(e), was still a typical NLP.

Here, we would like to mention another point regarding the generation of SRs in fiber lasers. SRs have been reported in various cavity designs, such as unidirectional ring cavities [26, 27] and figure-eight cavities [24]. To the best of our knowledge our fiber laser has the longest cavity length. However, it is difficult to conclude whether it is better or not to use a very long fiber cavity for the generation of SRs.

\section{MECHANISMS RELATING TO SCALAR SRS}

As for the physical mechanisms behind the SRs shedding from the NLP, the dramatically-enhanced local nonlinearity due to the MKR may be a partial answer. The diameter of the fiber taper is only $1.57 \mu \mathrm{m}$, noted as $d_{\text {taper }}$, about $1.256 \%$ of that of the original fiber. Considering that, for so thin a taper, the propagating optical field might fill the whole cross section, thus we should calculate the nonlinearity using the whole cross section area, rather than only using the tapered core area. The nonlinear parameter $\gamma$ of a telecommunication SMF at $1.55 \mu \mathrm{m}$ wavelength is typically $2 \mathrm{~W}^{-1} \mathrm{~km}^{-1}$ [28]. As for the tapered fiber, $\gamma$ can be calculated by using the following relationship [29]

$$
\gamma=\frac{8 n_{2}}{\lambda d_{\text {taper }}^{2}}\left(1-\frac{1}{V^{4}}\right) \ln V
$$

where $\lambda$ is the central wavelength and $n_{2}$ is the fiber nonlinearindex coefficient that can be calculated through [30]

$$
n_{2} \approx\left[2.507+25.25\left(N A / n_{\text {silica }}\right)^{2}\right] \times 10^{-20} \mathrm{~m}^{2} / \mathrm{W} \text {. (2) }
$$

In it, $n_{\text {silica }} \approx 1.444$ near $1.55 \mu \mathrm{m}$ wavelength, and $N A$ is the numerical aperture. For a fiber taper, the refractive index of the surrounding air cladding is roughly 1 . Thus,

$$
N A \approx \sqrt{n_{\text {silica }}^{2}-1} \approx 1.042
$$

$n_{2}$ can then be calculated as $\sim 1.566 \times 10^{-19} \mathrm{~m}^{2} / \mathrm{W}$.

$V$ parameter of the fiber taper can be calculated via

$$
V \approx \frac{\pi d_{\text {taper }}}{\lambda} \sqrt{n_{\text {silica }}^{2}-1} \text {. }
$$

Thus, $V \approx 3.315$.

Finally, the calculated $\gamma \approx 387.2 \mathrm{~W}^{-1} \mathrm{~km}^{-1}$, which enhanced by $\sim 193.6$ times in comparison to the original fiber before tapering. Thus, the MKR results in dramatic enhancement of the local nonlinearity even for a single circulation in the MKR. Further considering the resonant properties of the MKR, the optical pulse can circulate within the MKR for numerous loopthe-loops before it continues to complete a whole cavity roundtrip. Thus, the resulted locally nonlinear effects can experience considerable magnifications.

Benefiting from these, the detailed formation of the SRs can be analyzed as follows. When the pump power was increased to beyond $\sim 164.8 \mathrm{~mW}$, the peak power of the intra-pulse of the NLP was strong enough to trigger the obvious spectrum broadening. At the same time, the dense spectral modulation of the MKR limited the spectrum broadening and instead favored the multiple soliton generation. As the multiple solitons were originated from NLPs, they naturally inhibited instability which behaves as soliton rains. Further pump increase resulted in more soliton shedding, that is, expanding of the temporal span of the SRs.

Besides aforementioned mechanisms attributed to the use of MKR, other possible cause for the formation of SRs through partially depleting the NLP might be the condensed phase 
achieved by the extension and drift of strong continuous wave (CW) noise background [23]. As previously noted in Figs. 4(a) and $4(\mathrm{~b})$, the soliton rain appeared only when a spectral peak started to standing out from the main spectral profile. This spectral peak was in fact due to some CW components. Despite the spectrally identified CW noise background, the pedestal in Fig. 4(d) covering the whole span of the used autocorrelator should be another indicator.

The physical mechanism for the generated SRs only along one polarization direction should attribute to the polarization dependent loss of the fabricated MKR. As seen in Fig. 4(a), the amplitude of the vertical NLP was higher than that of the horizontal one, whereas they exhibited roughly equal durations. Further taking into account that the shedding SRs also in the vertical direction, the overall amount of the vertically distributed pulse energy was much greater than that with horizontal distribution. These two points indicate that the used MKR indeed has polarization-dependent loss, and the SRs tend to be formed in the polarization direction with less loss. After numerous shaping effects in the MKR, only the SRs in a single polarization, where the MKR has the least loss, can be left, becoming scalars.

Another noted phenomenon is that, although all the SRs are reduced to scalars, the NLP still remains vector characteristics. This is also understandable. It is well known that, in comparison to high peak pulses, the $\mathrm{CW}$ or low peak power pulses always experience stronger depletion in fiber lasers when a saturableabsorbing device is incorporated. Here, the NALM acts as the saturable absorber. Thus, considering that the peak power of the formed SRs is indeed lower than that of the NLP, seen in the upper part of Fig. 4(a), and also considering that the transmission loss of the MKR is polarization-dependent, the SRs would preferably appear in the polarization direction that has lower loss. In Fig. 4, it is noted as along the vertical axis that the MKR has the smallest loss. In other polarization directions, the SRs become weaker, and reach the weakest when the polarization direction is orthogonal. From this point of view, the MKR can be also considered as a polarization-dependent absorber. Thus, after repeatedly circulating and reshaping in the MKR, finally the SRs will only appear in the polarization where the transmission loss is the least, i.e., the SRs consequently become scalars. Whereas, due to the higher peak power of the internal sub-pulses of the NLP and the wide pulse duration of the NLP with the random evolution, the polarization-dependent absorption difference would be much weaker. This results in the survival of NLPs along the two orthogonal polarization directions. Thus, the NLP cannot be reduced to a scalar, instead remaining the vector characteristics.

\section{CONCLUSIONS}

In conclusion, we have experimentally realized scalar SRs shedding away from a vector NLP regime by introducing an MKR device into an ultralong EDF laser. Manipulation on the temporal dynamics was achieved by using the MKR through the spectral tailoring and local nonlinearity management, which potentially paves new ways for the applications of MKR in fiber lasers.

\section{ACKNOWLEDGEMENT}

Lei Li and Luming Zhao would like to thank the Jiangsu Overseas Visiting Scholar Program for University Prominent Young MiddleAged Teachers.

\section{REFERENCES}

[1] A. Autere, H. Jussila, Y. Dai, Y. Wang, H. Lipsanen, Z. Sun, "Nonlinear optics with 2D layered materials," Adv. Mater., vol. 30, no. 24, pp. 1705963, 2018.

[2] B. Guo, Q. Xiao, S. Wang, H. Zhang, "2D layered materials: synthesis, nonlinear optical properties, and device applications," Laser Photon. Rev., vol. 13, no. 12, pp. 1800327, 2019.

[3] C. S. Jun, S. Y. Choi, F. Rotermund, B. Y. Kim, and D. Yeom, "Toward higher-order passive harmonic mode-locking of a soliton fiber laser," Opt. Lett., vol. 37, no. 11, pp. 1862-1864, 2012.

[4] W. H. Renninger, A. Chong, and F. W. Wise, "Area theorem and energy quantization for dissipative optical solitons," J. Opt. Soc. Am. B, vol. 27, no. 10, pp. 1978-1982, 2010.

[5] A. Chong, W. H. Renninger, and F. W. Wise, "All-normal-dispersion femtosecond fiber laser with pulse energy above $20 \mathrm{~nJ}$," Opt. Lett., vol. 32, no. 16, pp. 2408-2410, 2007.

[6] M. Tang, H. Wang, R. Becheker, J. L. Oudar, D. Gaponov, T. Godin, and A. Hideur, "High-energy dissipative solitons generation from a large normal dispersion Er-fiber laser," Opt. Lett., vol. 40, no. 7, pp. 14141417, 2015.

[7] W. Chang, A. Ankiewicz, J. M. Soto-Crespo, and N. Akhmediev, "Dissipative soliton resonances," Phys. Rev. A, vol. 78, no. 2, pp. 023830, 2008.

[8] X. Wu, D. Y. Tang, H. Zhang, and L. M. Zhao, Opt. Express, "Dissipative soliton resonance in an all-normal-dispersion erbium-doped fiber laser," vol. 17, no. 7, pp. 5580-5584, 2009.

[9] S. K. Wang, Q. Y. Ning, A. P. Luo, Z. B. Lin, Z. C. Luo, and W. C. Xu, "Dissipative soliton resonance in a passively mode-locked figure-eight fiber laser," Opt. Express, vol. 21, no. 2, 2402-2407, 2013.

[10] H. Liu, X. Zheng, N. Zhao, Q. Ning, M. Liu, Z. Luo, A. Luo, and W. $\mathrm{Xu}$, "Generation of multiwavelength noise-like square-pulses in a fiber laser," IEEE Photon. Technol. Lett., vol. 26, no. 19, pp. 1990 - 1993, 2014.

[11] J. Liu, Y. Chen, P. Tang, C. Xu, C. Zhao, H. Zhang, and S. Wen, "Generation and evolution of mode-locked noise-like square-wave pulses in a large-anomalous-dispersion Er-doped ring fiber laser," Opt. Express, vol. 23, no. 5, pp. 6418-6427, 2015.

[12] Z. Deng, G. Zhao, J. Yuan, J. Lin, H. Chen, H. Liu, A. Luo, H. Cui, Z. Luo, and W. Xu, "Switchable generation of rectangular noise-like pulse and dissipative soliton resonance in a fiber laser," Opt. Lett., vol. 42, no. 21, pp. 4517-4520, 2017.

[13] Y. Huang, Y. Qi, Z. Luo, A. Luo, and W. Xu, "Versatile patterns of multiple rectangular noise-like pulses in a fiber laser," Opt. Express, vol. 24, no.7, pp. 7356-7363, 2016.

[14] Y. Huang, Z. Hu, H. Cui, Z. Luo, A. Luo, and W. Xu, "Coexistence of harmonic soliton molecules and rectangular noise-like pulses in a figureeight fiber laser," Opt. Lett., vol. 41, no.17, pp. 4056-4059, 2016.

[15] B. Guo, Y. Yao, Y. Yang, Y. Yuan, L. Jin, B. Yan, and J. Zhang, "Dualwavelength rectangular pulse erbium-doped fiber laser based on topological insulator saturable absorber," Photon. Res., vol. 3, no.3, pp. 94-99, 2015.

[16] X. Jiang et al., "Demonstration of optical microfiber knot resonators," Appl. Phys. Lett., vol. 88, Art. no. 1380, 2006.

[17] M. Sumetsky, Y. Dulashko, J. M. Fini, A. Hale, and D. J. DiGiovanni, "The microfiber loop resonator: theory, experiment, and application," J. Lightwave Technol., vol. 24, no. 1, pp. 242-250, 2006.

[18] G. Brambilla, F. Xu, P. Horak, Y. Jung, F. Koizumi, N. P. Sessions, E. Koukharenko, X. Feng, G. S. Murugan, J. S. Wilkinson, and D. J. Richardson, "Optical fiber nanowires and microwires: fabrication and applications," Adv. Opt. Photon., vol. 1, no. 1, pp. 107-161, 2009.

[19] Z. Chen, V. K. S. Hsiao, X. Li, Z. Li, J. Yu, and J. Zhang, "Optically tunable microfiber-knot resonator," Opt. Express, vol. 19, no. 15, pp. 14217-14222, 2011. 
[20] M. Liu, R. Tang, A. Luo, W. Xu, and Z. Luo, "Graphene-decorated microfiber knot as a broadband resonator for ultrahigh-repetition-rate pulse fiber lasers," Photon. Res., vol. 6, no. 10, pp. C1-C7, 2018.

[21] S. Li, Y. Yi, Y. Yin, Y. Jiang, H. Zhao, Y. Du, Y. Chen, E. Lewis, G. Farrell, S. W. Harun, and P. Wang, "A microfiber knot incorporating a Tungsten Disulfide saturable absorber based multi-wavelength modelocked erbium-doped fiber laser," J. Lightwave Technol., vol. 36, no. 23, pp. 5633-5639, 2018.

[22] J. Zhou, Z. Chen, and J. Zhao, "Microfiber-Knot-Resonator-Induced Partial Elimination of Longitudinal Modes in Fiber Lasers for In-TuneSwitchable Nanosecond Pulse Generation," J. Lightwave Technol., vol. 38 , no. 4, 875-880, 2020 .

[23] S. Chouli and P. Grelu, "Rains of solitons in a fiber laser," Opt. Express, vol. 17, no.14, pp. 11776-11781, 2009

[24] A. Niang, F. Amrani, M. Salhi, P. Grelu, and F. Sanchez, "Rains of solitons in a figure-of-eight passively mode-locked fiber laser," Appl. Phys. B, vol. 116, no. 3, pp. 771-775, 2014.

[25] M. Liu, A. Luo, X. Zheng, N. Zhao, H. Liu, Z. Luo, W. Xu, Y. Chen, C. Zhao, and H. Zhang, "Microfiber-based highly nonlinear topological insulator photonic device for the formation of versatile multi-soliton patterns in a fiber laser," J. Lightwave Technol., vol. 33, no. 10, pp. 2056-2061, 2015.

[26] S. Chouli, and P. Grelu, "Soliton rains in a fiber laser: An experimental study," Phys. Rev. A, vol. 81, no. 14, pp. 063829, 2010.

[27] S.S. Huang, Y.G. Wang, P.G. Yan, G.L. Zhang, J.Q. Zhao, H.Q. Li, and R.Y. Lin, "Soliton rains in a graphene oxide passively mode-locked ytterbium doped fiber laser with all normal dispersion," Laser Phys. Lett., vol. 11, pp. 025102, 2014.

[28] G. P. Agrawal, Nonlinear Fiber Optics, 5th ed. Amsterdam, The Netherlands: Elsevier, 2013.

[29] A. Zheltikov, "Gaussian-mode analysis of waveguide-enhanced Kerrtype nonlinearity of optical fibers and photonic wires," J. Opt. Soc. Am. B, vol. 22, no. 5, pp. 1100-1104, 2005.

[30] J. Zhao, L. Li, L. Zhao, D. Tang, and D. Shen, "Cavity-birefringencedependent h-shaped pulse generation in a thulium-holmium doped fiber laser," Opt. Lett., vol. 43, no. 2, 247-250, 2018.

[31] T. A. Birks, W. J. Wadsworth, and P. S. J. Russell, "Supercontinuum generation in tapered fibers," Opt. Lett., vol. 25, no. 19, pp. 1415-1417, 2000 .

Jian Zhou is currently pursuing the Master's Degree at the Jiangsu Key Laboratory of Advanced Laser Materials and Devices, School of Physics and Electronic Engineering, Jiangsu Normal University, Xuzhou, China. He is mainly studying on mode-locked fiber lasers, fiber amplifiers, and particular microfiber devices.

Shuchang Gu is an undergraduate student in Physics, School of Physics and Electronic Engineering, Jiangsu Normal University, Xuzhou, China. She mainly participates the fabrication of fiber tapers.

Zikai Chen is an undergraduate student in Physics, CW Chu College, Jiangsu Normal University, Xuzhou, China. He mainly participates the fabrication of various microfiber devices.

Bowen Lou is currently pursuing the Master's Degree at the Jiangsu Key Laboratory of Advanced Laser Materials and Devices, School of Physics and Electronic Engineering, Jiangsu Normal University, Xuzhou, China. He is mainly studying on the dynamics in ultrafast fiber lasers.

Yuyuan Jiang is currently pursuing the Master's Degree at the Jiangsu Key Laboratory of Advanced Laser Materials and Devices, School of Physics and Electronic Engineering, Jiangsu Normal University. She is mainly studying on fiber-based devices and systems.

Junqing Zhao received the Doctor of Engineering degree in Optical Engineering from Shenzhen University, Shenzhen, China, in 2014, for research on pulsed fiber lasers. Since then, his research has covered device, system, and application aspects of fiber lasers and amplifiers, first with the Shenzhen Key Laboratory of Laser Engineering, Shenzhen University, China, and later with the Optoelectronics Research Centre (ORC), University of Southampton, U.K. He is currently with the Jiangsu Key Laboratory of
Advanced Laser Materials and Devices, School of Physics and Electronic Engineering, Jiangsu Normal University, Xuzhou, China. He has published some 50 scientific articles. He is a Life Member of the Optical Society of America.

Lei Li was born in 1983. He received his bachelor and master degree in optics from Shandong Normal University, Jinan, China. He received his Ph.D degree in optical engineering from Shandong University, Jinan, China, in 2013. His research filed focuses on the ultrafast fiber lasers, nonlinear optics and optoelectronics technology.

Dingyuan Tang received the B.S. degree in physics from Wuhan University, in 1983, the M.S. degree in laser physics from the Shanghai Institute of Optics and Fine Mechanics, Chinese Academy of Science, in 1986, and the Ph.D. degree in physics from the Hannover University, Germany, in 1993. From 1993 to 1994, he worked as a scientific employee at the PhysikalischTechnische Budesanstalt, Braunschweig, Germany. From 1994 to 1997, he was a university Postdoctoral Research Fellow, and from 1997 to 1999, he was an Australian Research Council (ARC) Postdoctoral Research Fellow, both at the University of Queensland, Australia. From 1999 to 2000, he was a Research Fellow in the Optical Fiber Technology Center (OFTC), the University of Sydney, Australia. He is currently an Associate Professor in the School of Electrical and Electronic Engineering, Nanyang Technological University, Singapore, and a Special Professor at Jiangsu Normal University, China. Dr. Tang is a Member of Optical Society of America (OSA) and the Australian Optical Society (AOS).

Deyuan Shen received the Ph.D. degree in physics from Shandong University, Jinan, China, in 1996. He subsequently joined the Institute for Laser Science, University of Electro-Communications, Tokyo, Japan, as a Postdoctoral Research Fellow, where he worked on laser-diode pumped highpower solid-state lasers. From 2000 to 2002, he was with the School of Electrical, and Electronic Engineering, Nanyang Technological University, Singapore, developing high-power visible and infrared solid-state lasers for industrial applications. He joined the Optoelectronics Research Centre (ORC), University of Southampton, Southampton, U.K., in 2002, and promoted to a Senior Research Fellow in 2005. His research activities at ORC included eyesafe high-power fiber lasers, fiber-bulk hybrid solid-state lasers, and novel fiber laser sources. His current research interests include power and brightness scaling of cladding pumped fiber lasers and amplifiers, nonlinear frequency conversion, and laser applications.

Lei Su received the B.Eng. and M.Eng. degrees from Xi'an Jiaotong University, Xi' an, China, and the Ph.D. degree from Nanyang Technological University, Singapore. He is a Lecturer in biomedical engineering with the School of Engineering and Materials Science, Queen Mary University of London, London, U.K.

Luming Zhao received the B.Eng., and M.Eng. degrees from Tsinghua University, Beijing, China, and the Ph.D. degree from Nanyang Technological University, Singapore. He is currently a Full Professor with Jiangsu Normal University, Xuzhou, China. His research interests include ultrafast optics, fiber oscillators, fiber amplifiers, and soliton dynamics. 